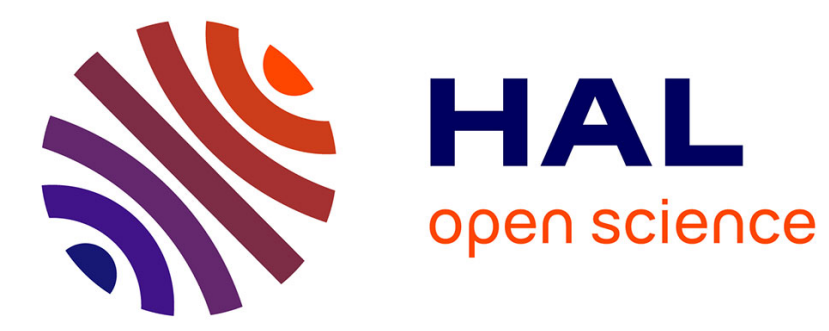

\title{
Go Mapping Theory and Factor Space Theory Part I: An Outline
}

He Ouyang

\section{To cite this version:}

He Ouyang. Go Mapping Theory and Factor Space Theory Part I: An Outline. 2nd International Conference on Intelligence Science (ICIS), Oct 2017, Shanghai, China. pp.37-41, 10.1007/978-3-31968121-4_4. hal-01820908

\section{HAL Id: hal-01820908 https://hal.inria.fr/hal-01820908}

Submitted on 22 Jun 2018

HAL is a multi-disciplinary open access archive for the deposit and dissemination of scientific research documents, whether they are published or not. The documents may come from teaching and research institutions in France or abroad, or from public or private research centers.
L'archive ouverte pluridisciplinaire HAL, est destinée au dépôt et à la diffusion de documents scientifiques de niveau recherche, publiés ou non, émanant des établissements d'enseignement et de recherche français ou étrangers, des laboratoires publics ou privés. 


\title{
Go Mapping Theory and Factor Space Theory Part I: An Outline
}

\author{
He Ouyang \\ Sunbridge Grothendieck Institute \\ Dept. of Mathematics, School of Science, National University of Defense Technology. \\ Changsha, Hunan, PRC.
}

\begin{abstract}
Inspired by Professor Wang, Peizhuang's Factor Space Theory(FST), we propose a new scheme, called GO Mapping Theory, or GMT in short, to formalize the concept formation knowledge representation of AI. This scheme can be viewed as an extension of Willie's Formal Concept Analysis(FCA), PZ Wang's Factor Space Theory, and it naturally includes Gouguen's L-Fuzzy Sets therefore it sounds a unified soft computing scheme. Potentially, GMT can be used for human-like knowledge representation and computation by modern computers. By deploying Grothendieck's topos theory (this is the origin for the name GO mapping), we developed a unified mathematical language understandable by robots which can represent human language: concepts and logic, which also unified the current learning techniques at a more abstract level, therefore can be used as a basis for AGI or Super AI.

By restating FST under category language, one can have a much more general setup for classical reductionist's view about multiple sensory system, we call it a cognitive frame. Then under the assumption of uncertainty of any measurement, we can naturally, in fact ontologically, obtain an L-fuzzy set by FST, then we can construct from this L-fuzzy set a L-presheaf by standard procedure, we call it the GO mapping, which by Barr's Embedding Theorem, can be viewed as the natural replacement for classical fuzzy sets. In fact, in topos theory, FST and GMT pairs a geometric morphism in Grothendieck's topos theory, which shows the amazing power of pure math in real life applications.
\end{abstract}

\section{Factor Space Theory (FST)}

Introduced by Prof Wang, PZ around 80's, [4], we have

Definition 1. Let $U$ be a set, called the universe, $f: U \rightarrow V_{f}$ is a map, we call $f$ a factor for $U$. If $\left\{f: U \rightarrow V_{f}\right\}$ is a set of factors, we call $S S=\prod V_{f}$ the state space and $F: U \rightarrow S S$ the factor space.

The basic idea is this: when we want to know $U$, or an object in $U$, we try to use several "detectors", or "sensors/measurements/instruments", to gauge/measure $U$, we call these detectors "the factors" (by Wang). This is in fact the reductionist / instrumentalists idea. 
Afterwards, we try to analyze the result in the state space to see, to find out where these values falling. And from there, one can do logic operations and draw conclusions and extract information about $U$.

In this paper, we will assume all sets are topological spaces and we would like to modify the definition 1 slightly as follows:

Before giving the definition, let's recall some terminologies from category theory.

Definition 2. A diagram $\mathcal{D}$ in a category $\mathfrak{C}$ is a collection of objects as vertices and some morphisms among these objects as edges.

$A$ cone $X$ is for a diagram $\mathcal{D}$ in $\mathfrak{C}$ is a collection of arrows $f_{i}: X \rightarrow D_{i}$ such that for any $g: D_{i} \rightarrow D_{j}$ in the diagram $\mathcal{D}$, following diagram

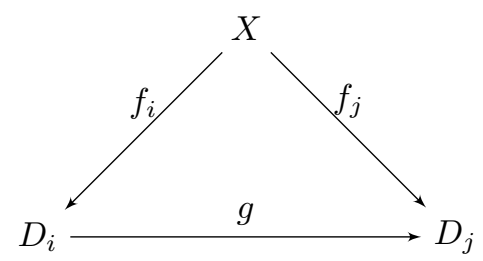

commutes. Cone is usually denoted by $\left\{f_{i}: X \rightarrow D_{i}\right\}=$ Cone. Reference [?] for details.

This way, we can rewrite the factor space theory as follows.

From cognitive point of view, we can identify a cone as the most general format of factor space theory. Or we can view a cone as a cognitive frame for object $X$. $\mathcal{D}$ can be viewed the collection of instruments.

Let's remind what is the limit for a diagram. In a short sentence, the limit is a universal object satisfying

1. It is a cone for $\mathcal{D}$, denoted by $\lim \mathcal{D}$

2 . It is a "minimum" cone among all cones over $\mathcal{D}$

which means for any cone $K$ over $\mathcal{D}, \exists$ ! arrow $F: K \rightarrow \lim \mathcal{D}$ such that

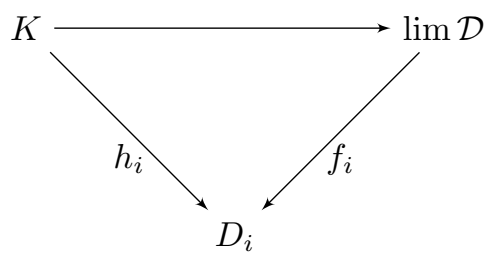

commutes.

After these preparation, we can give a more elegant definition for Factor Space Theory.

Definition 3. Let Top be the category with topological spaces as objects and continuous maps as arrows. Then any diagram in Top has the limit. We call any cone $\left\{f: U \rightarrow V_{f}\right\}$ in Top the cognitive frame for the universe $U$

We call $\lim V=\lim \left\{V_{f}\right\}$ the state space and the unique map $F: U \rightarrow \lim V$ the cognitive map. 


\section{Formal Concept Analysis (FCA)}

In 80's, Wille developed a concept called Formal Concept Analysis, FCA in short. FCA has gained great applications in computer science.

In FCA, $\forall U, V \in$ Sets, $F \subset U \times V$, the triple $(U, F, V)$ is called a formal context, if $A \subset U, B \subset V$ satisfy

$$
F A=B, \quad F^{-1} B=A
$$

We call $A$ and $B$ are Galois connected and $(A, F, B)$ a formal concept.

This theory in some sense encodes the classic concept in classic logic for computer science. It is easily identified that set $A$ is the extension for a concept, and $B$ is the intension for this concept. Obviously, FCA $(A, F, B)$ has best captured the classical usage of concepts for bots, if human mind can be determined by Boolean logic. In the work of [?], we prove that under definition 3, we have

\section{Theorem 1.}

$$
F S T \supset F C A
$$

Hence, from section 1, we know that one cognitive from theory (CFT) is the most general set operation:

$$
C F T \supset F S T \supset F C A
$$

\section{Gouguen's L-fuzzy sets and Barr's Embedding}

Let $H$ be a Heytin algebra. Then Gouguen introduced a category of H-fuzzy sets by define:

$-\operatorname{Obj}(\operatorname{Fuz}(H))=\{x: X \rightarrow H, X \in \operatorname{Sets}\}$

- Arrows $f:(X, x) \rightarrow(Y, \eta) \Leftrightarrow f: X \rightarrow Y$ in Sets and $x \leq \eta \circ f: X \rightarrow H$

Unfortunately, $\operatorname{Fuz}(H)$ is not a topos except $H$ is a Boolean algebra. The great advantage for working math, AI, mind computing is that Topos behaves almost like Sets, except the law of excluding the middle (LEM) .

In real life logic, LEM probably the most wrong law human follows, hence it is born for abandoning.

However, to proceed the basic logic deduction, subsets, power sets, produts, limit, colimit and all basic ingredients. Topos provide all these needed goodies.

1980s Michael Barr[3] \& Pitts both showed that the Gouguen category is not a topos, but can be embedded in some way into a topos. Here we will give a short description on Barr's construction.

$\forall \alpha \in H$, we can define a "level" set $X_{\alpha}=\{y \in X: x(y) \geq \alpha\}$, then we call the collection $\mathcal{T}(X)=\tilde{X}=\left\{X_{\alpha}: \alpha \in H\right\}$ the tow of $X$. One can prove that $\mathcal{T}: \operatorname{Fuz}(H) \rightarrow \operatorname{Tow}(H)$ is an isomorphism of categories. Each element in fact looks like $G: H \rightarrow$ Sets satisfying the axioms for presheaf, i.e. $G$ is 
a contravariant functor from partial order set considered as a category to the category set.

The Barr proved by providing an initial element $z$ to $H$, one can "embed" the cat $\operatorname{Tow}(H)$ into a topos $\operatorname{Sh}\left(H^{+}\right)$, where $H^{+}=H \cup\{z\}, \quad z<a \quad \forall a \in H$ and $\operatorname{Sh}\left(H^{+}\right)$is the category of $H^{+}$-sheaves.

This is a very significant result. Because one can translate every "fuzzy" math problem into a problem about sheaf/presheaf then using the powerful tool sets since Grothendieck developed algebraic geometry.

This is analogue to embedding the rationals to reals!

From Barr and Pitts, we in fact showed how to consider every presheaf

$$
G: H \rightarrow \text { Sets }
$$

to be some sort of "fuzzy sets" or "fuzzy concepts".

Using presheaves directly instead of the Zadeh/Gouguen fuzzy sets will provide powerful tools for soft computing \& AI, from our perspective.

\section{The GO Mapping Theory (GMT)}

In general in a real world, any measurement can be viewed as a function

$$
f: U \rightarrow V
$$

Normally, $V$ is a finitely dimensional vector space equipped with a positive metric $C$, most possibly a metric/a topology induced by an $L^{P}$-norm, $p=1,2, \infty$ would be the most popular ones. Then people will define/conceptualize a thing based on the values of the measurement. This is established since Aristotle's era and in 1980's formalised by FST, FCA and Feng's Property Mapping Theory (PMT).

Here we will develop a new scheme which can include all the theories above as a special case and naturally incorporate the intrinsic fuzziness of measurements and human mind computation.

Let's go back to the example above.

In reality, no measurement is precise (in face we do not have a god given correct precise value for any), hence in most cases what we know is that $\forall u \in U$, $f(u)$ is some neighborhood of a center value $x \in V$, i.e. $f(u) \in B(x)$ This way, one is more interested in $f^{-1}(B) \subset U$. for some open set $B$. Hence $f$ induces a map

$$
G: \mathscr{O}(V) \rightarrow \mathscr{P}(U)
$$

by "defining"

$$
G(B)=f^{-1}(B)
$$

where $\mathscr{O}(V)$ is the collection of open sets of $V$. From [2], [1], we know that any $f: U \rightarrow V$ induces a geometric morphism

- the direct image $f_{*}: P S h(U) \rightarrow P S h(V)$

- the inverse image $f^{*}: P S h(V) \rightarrow P S h(U)$ 
where $P S h$ stands for the topos of presheaves.

This way, we can construct the GO mapping for a cognitive frame as follows: Let $F: U \rightarrow V=\lim V_{f}$ be the factor mapping. $\forall s \in P S h(U)$, We will call

$$
F^{*} \circ s: \mathscr{O}(V) \rightarrow \text { Sets }
$$

a GO mapping by "Fuzzy" set $s$. In fact, $\forall B \in \mathscr{O}(V), F^{*} \circ s(B)=s\left(F^{-1}(B)\right)$. Sometime we also call the functor $F^{*}$ the GO mapping, denote it by $G$.

\section{References}

1. Johnstone, Peter, Topos Theory

2. Johnstone, Peter, Sketches of an Elephant: A Topos Theory Compendium

3. Barr, M., fuzzy set theory and topos theory

4. Wang, Peizhuang, Factor Space, the Theoretical Base of Data Science 